\title{
A Collaborative Approach for Segmentation of Probe Image for Efficient Texture Recognition
}

\author{
Divya Mathur \\ Department of Computer Science \& Engineering \\ Techno India NJR Institute of Technology, Udaipur
}

\author{
Sandeep Upadhyay \\ Department of Computer Science \& Engineering \\ Techno India NJR Institute of Technology, Udaipur
}

\begin{abstract}
Image processing methodologies and domain is quite wide and really efficient now days for real time applications. Our work primarily deals with the domain of image segmentation and using segmentation concept, texture recognition has been performed with comparative results and simulations performed over a particular image dataset. The initial work in our proposed work is to perform segmentation on each part image then performing extraction. We have focused on segmentation followed by extraction so that the classification result may not contain much error. The conventional approach has been implemented in this regard first and then the main problem that has been formulated is patch up data pixels together which provide error in getting right and appropriate texture .In order to deal with the problem formulated in the existing work we have proposed a new commuted method in which the extraction and segmentation of image depends on the dynamic threshold set by user.
\end{abstract}

\section{Keywords}

Weka, Naïve Bayesian

\section{INTRODUCTION}

The world as we all know nowadays is extremely fast paced and extremely machine-driven. Technology has become similar to automation. All this is often as a result of we have a tendency to, humans, have a bent to try to to our job quicker and within the most effective method. thus the additional we have a tendency to change, the simpler and quicker our work becomes. Next trend in today's quick dynamical world is medical care. Since this is often the age of computers we would like each data obtainable be digitized and keep within the computers, since they need quicker computing capabilities. however the matter in digitizing world data into digital domain is we'd like to show the pc specifically concerning our involved world information.

Texture affirmation (OCR) is the distinction in physically formed or created substance into an electronic course of action, which can be secured, deciphered and orchestrated by a PC. It can be utilized as a snappy information input framework for a front line PC. Any OCR framework depends upon the running with four key advances:- Texture affirmation (OCR) is the difference in translated or created substance into an electronic plan, which can be secured, deciphered and dealt with by a PC. It can be utilized as a provoke information input procedure for a front line PC. Any OCR framework depends upon the running with four key advances:-.

\section{BACKGROUND}

in certainties, principal Component assessment (pca) can be a technique that might be acclimated change a dataset., extra formally it's far a straight change that picks a front line reference outline for the informational collection specific the fine fluctuation through any projection of the actualities set incorporates lie on the essential hub (at that point called the essential most critical segment), the second quality difference on the second one pivot, et cetera. PCA are often utilized for diminishing dimensionality in a greatly dataset while retentive those attributes of the dataset that make a commitment most to its variance with the guide of expelling the later imperative components (with the asset of an extra or a dreadful parcel considerably less heuristic decision). Those qualities additionally can be the "greatest basic", however this can be not essentially the situation, depending on the applying.

PCA has the forte of being the top notch straight change numerical space that has greatest fluctuation. In any case, this comes at the doubtlessly worth of vast approach call for. never again like probability direct changes, the PCA does not have an intense and fast arrangement of premise vectors, its premise vectors depend on the data set. expecting 0 exact propose (the observational suggest of the circulation has been subtracted from the information set), the fundamental part American joined conditions of a dataset $x$ are every now and again compute with the asset of finding the Eigen values and eigenvectors of the fluctuation lattice of $\mathrm{x}$, we discover that the eigenvectors with the most basic eigenvalues compare to the measurements which have the most effective correlation inside the dataset. the preparatory estimations ar eventually anticipated onto the diminished vector locale. Look based absolutely acknowledgment exploitation PCA

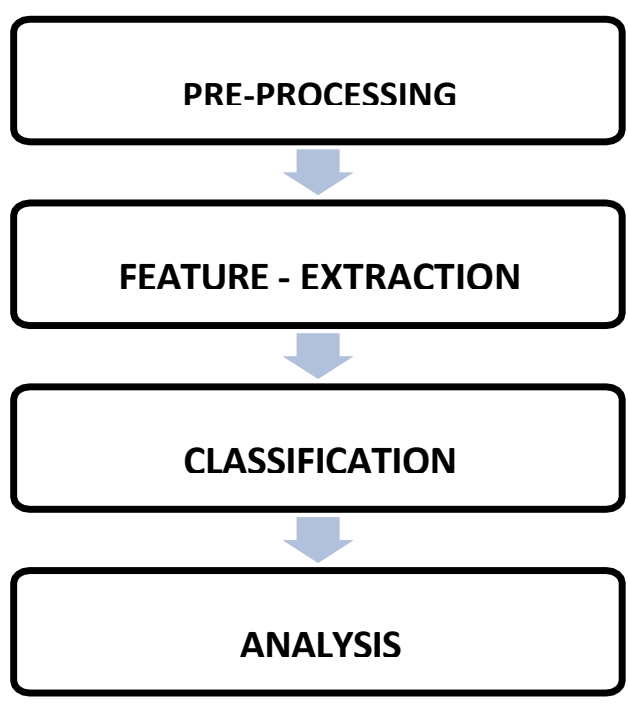

Figure 1.1: Steps for OCR

\section{PROBLEM FORMULATION AND PROPOSED SOLUTION}

In comparison of pervious work, our main focus would be on 3.2Proposed Algorithm

Step1 - Take input data set in RGB texture and then perform linearization for making it compatible for the tool. 
Step2- Pre-processing of data set should be performed to implement masking and filtering for getting more accurate results

Step3-In order to get accurate image segmentation and extraction

Step4- A maximum threshold is set now for pixel intensity of 30 so that object lower than this pixel vector can be removed in order to provide better visual effects and less distortion in the dataset and texture

Step5-Again the linearization of image will be repeated to get the final noise removed image.

Step6-To provide dynamic dimension to our proposed algorithm we have calculated region attributes of image.

Step7-Region properties can be calculated using bounding index for better texture recognition.

Step8-Bounding box plotting is done now for getting exact position and edge color of the input data set.

Step9-Now final object extraction will be performed using pseudo code like

$\% \%$ Objects extractionfigure

for $\mathrm{n}=1: \mathrm{Ne}$ where $\mathrm{L}$ and $\mathrm{Ne}$ is label connected component

$[\mathrm{r}, \mathrm{c}]=$ find $(\mathrm{L}==\mathrm{n}) ; \mathrm{r}$ and $\mathrm{c}$ are rows and column feature vectors

$\mathrm{n} 1=$ imagen $(\min (\mathrm{r}): \max (\mathrm{r}), \min (\mathrm{c}): \max (\mathrm{c}))$; $\operatorname{maximumand}$ minimum feature vectors are calculated to extract each texture .n is the position of texture and region property attributes .

Step10-The above steps will be repeated till the last texture is not extracted efficiently.

Step11- exitr

\section{SIMULATION AND RESULTS}

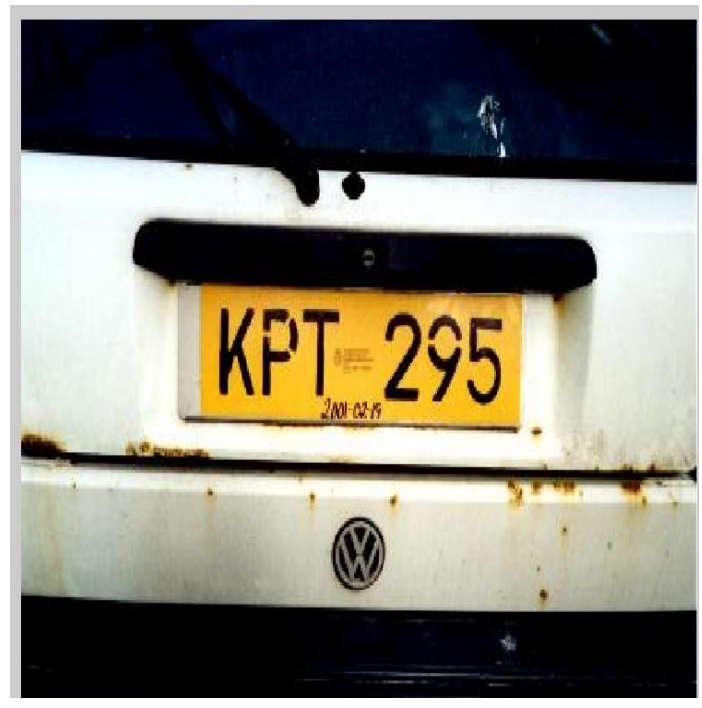

Fig 4.1 Input datalset of car number plate

The figure 1 is just used as in input data set which depicts the car number plate the first module will load this into the tool for preprocessing then the next process will be implemented the given image is used in implementing the existing work with random approach of setting the threshold value.

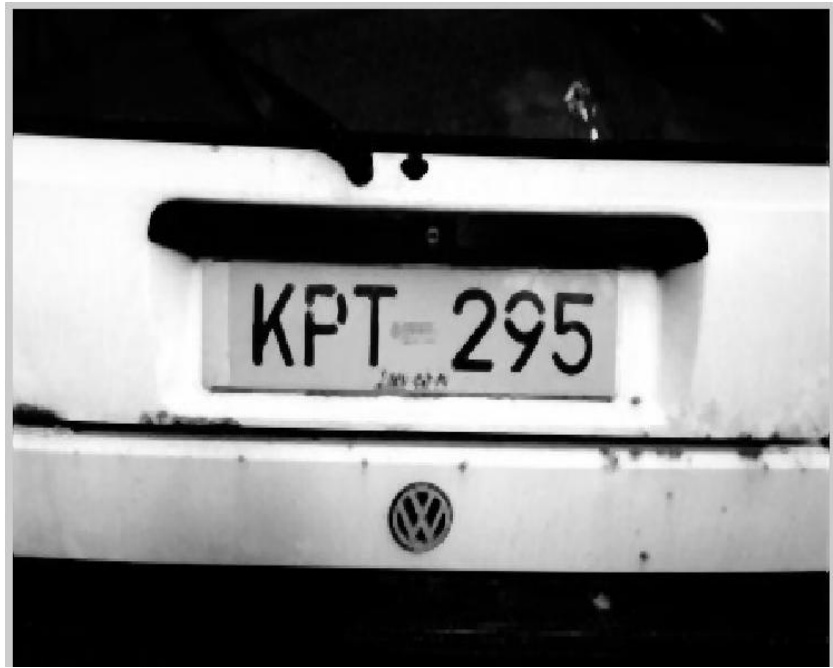

Fig 4.2.Binarization of the input data set

The fig 2 shown above is just the linearization of the input dataset so that it becomes compatible to the tool to get processed further.The 2 dimensional one is only acceptable in the tool for getting the number of maximum connected components so that we can get that number reduce in the proposed method to get the accuracy improved along with better time complexity and better extraction capabilities. The existing work has randomly set the maximum threshold for the probe image to extract the textures more accurately in the first module of the work .considering the module1.We have contemplated a new technique with dynamic approach to get the characters on a console window with more accuracy and clarity. Instead of setting the threshold value randomly we have used bounding boxes in the work and proposed algorithm

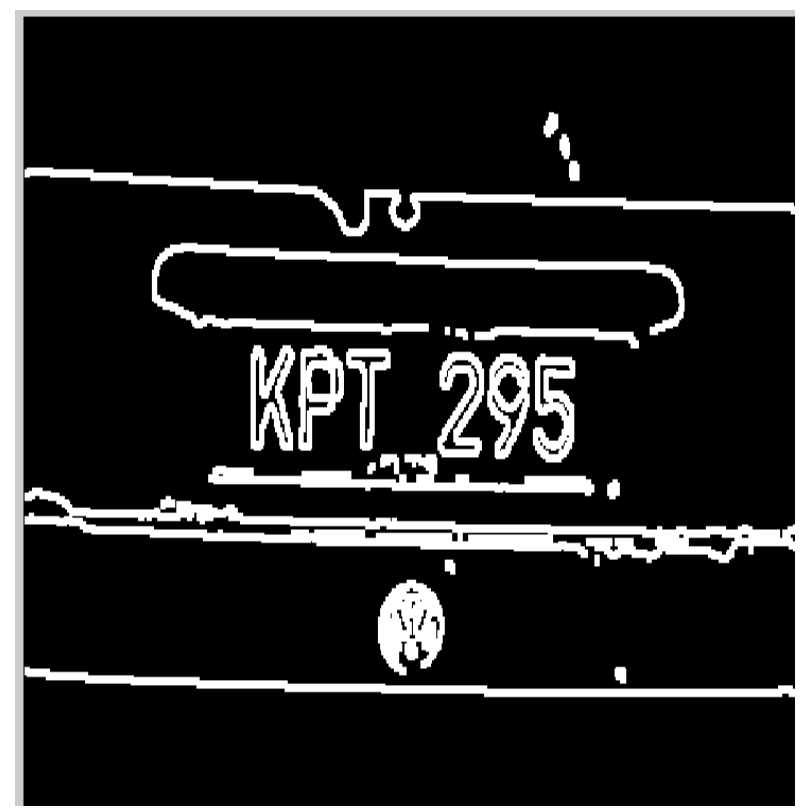

Fig 4.3.Texture segmentation of the probe image to get content separated

The fig 3 is showing the segmentation of different textures so that the content can be recognized accurately but the existing work has a drawback it can recognize s single character for each time, It will certainly increased the time complexity for each loop and it also requires us to set the random threshold for different character to be recognized. . The existing work has 
randomly set the maximum threshold for the probe image to extract the textures more accurately in the first module of the work .considering the module 1

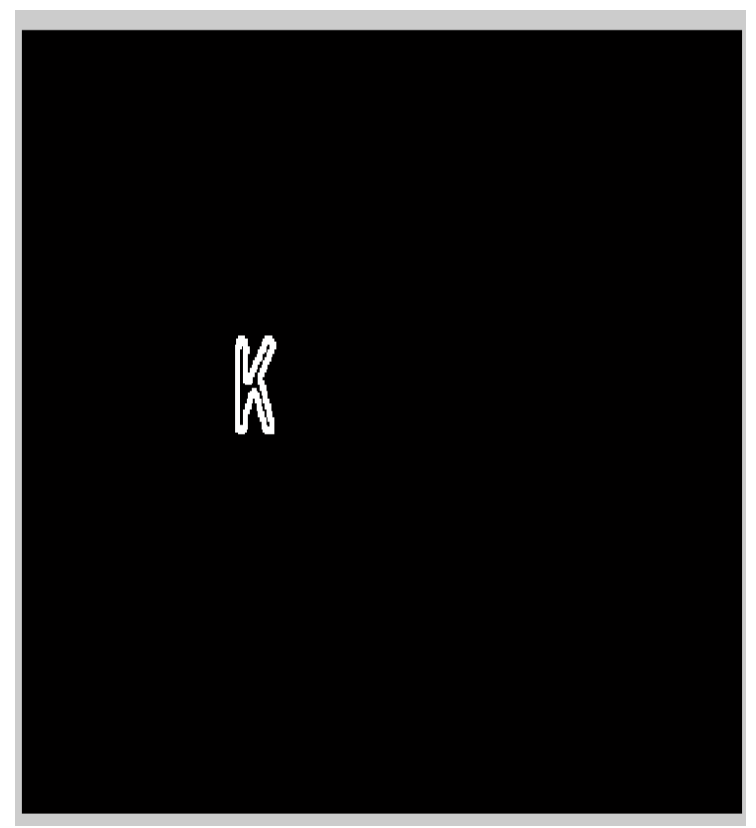

Fig.4.4.the single texture extracted character by using existing Algorithm

The Fig 4 has depicted the extracted texture which has been performed by using existing work algorithm which was very much random in approach so we can see that only single texture can be recognized at the single time this was one of the drawback of existing work

\section{CONCLUSION AND FUTURE WORK}

A road accident is something which misshapen with our Image processing methodologies and area is quite extensive and simply efficient now days for real time applications. There are different proposed approach which perform segmentation based texture recognition with on the web and disconnected methodologies with high and low accuracy of in recognition .Our work concentrates around commuted methodologies which incorporates pre-handling of test set first to show signs of improvement precision and afterward examine the output goes under the recognition task. The current work has picked irregular and static methodologies which at times blend information pixel and result in worst precision and poor texture recognition rate.

\section{REFERENCES}

[1] A. Chaudhuri et al., Texture Recognition Systems for Different Languages with Soft Computing, Studies in Fuzziness and Soft Computing 352, DOI 10.1007/978-3319-50252-6_2, Springer International Publishing AG 2017

[2] Debasish Basa ,Sukadev meher, "Handwritten Odia Character Recognition", Presented in the National Conference on Recent Advances in Microwave Tubes, Devices and Communication Systems, Jagannath Gupta Institute of Engineering and Technology, Jaipur, March 45,2011

[3] Rajean Plamondon, Fellow IEEE and Sargur N. Srihari ,Fellow IEEE , "On-Line and Off-Line Handwritting character Recognition: A Comprehensive Survey", 1EEE TRANSACTIONS ON PATTERN ANALYSIS AND MACHINE INTELLIGENCE. VOL. 22, NO. 1 JANUARY 2000

[4] G.Vamvakas, B.Gatos, N. Stamatopoulos, and S.J.Perantonis, "A Complete Texture Recognition Methodology for Historical Documents", the Eighth IAPR Workshop on Document Analysis Systems

[5] B.B. Chaudhuri U. Pal M. Mitra, "Automatic Recognition of Printed Oriya Script"

[6] Fumitaka KIMURA, Tetsushi WAKABAYASHI and Yasuji MIYAKE, “ On Feature Extraction for Limited Class Problem"

[7] Supriya Deshmukh, Leena Ragha, "Analysis of Directional Features - Stroke and Contour for Handwritten Character Recognition", 2009 IEEE International Advance Computing Conference (IACC 2009) Patiala, India, 6-7 March 2009

[8] M. Blumenstein, B. K. Verma and H. Basli, "A Novel Feature ExtractionTechnique for the Recognition of Segmented Handwritten Characters", 7th International Conference on Document Analysis and Recognition (ICDAR '03) Eddinburgh, Scotland: pp.137-141, 2003. 